\title{
Model Predictive Control of Energy Storage Systems for Power Tracking and Shaving in Distribution Grids
}

\author{
Alessandro Di Giorgio, Member, IEEE, Francesco Liberati, Member, IEEE, Andrea Lanna, \\ Antonio Pietrabissa, Francesco Delli Priscoli
}

\begin{abstract}
In this paper, a model predictive control (MPC) strategy is proposed to control the energy flows in a distribution network node (e.g. a distribution substation) equipped with an electric storage system (ESS) and serving a portion of the grid with high penetration of renewable energy sources (RES). The aim is to make the power flow at node level more controllable in spite of the presence of fluctuating distributed energy resources. In particular, the proposed control strategy is such that the controlled power flow at node level tracks the profile established on a day-ahead basis for efficient operation of the grid. That is achieved by letting the MPC controller decide the current storage power setpoint based on the forecasts of the demand and of the RES output. Theoretical results are reported on the stability of the proposed control scheme in a simplified setting foreseeing zero forecasting error. The performance of the system in the general case is then evaluated on a simulation basis. Simulations show the effectiveness in managing RES fluctuations in realistic settings.
\end{abstract}

Index Terms-Energy Storage System; Model Predictive Control; Renewable Energy Sources; Smart Grid.

\section{NOMENCLATURE}

$e^{p v} \quad$ Difference between the short term RES forecast and the day head one

$P^{l} \quad$ Bus active power demand

$P^{s} \quad$ Storage active power flow

$P^{p v} \quad$ Active power from photovoltaic plants

$P_{d a}^{p v} \quad$ Day ahead forecast of $P^{p v}$

$P_{s t}^{p v} \quad$ Short term forecast of $P^{p v}$

$P^{g} \quad$ Active power flow at HV/MV substation

$P_{d a}^{g} \quad$ Day ahead forecast of $P^{g}$

$P_{s t}^{g} \quad$ Short term forecast of $P^{g}$

$x \quad$ Storage state of charge

$\tilde{x} \quad$ Difference between the state of charge and the reference state of charge

$T \quad$ Sampling time

$N \quad$ Length of the MPC control window (time slots)

Symbol denoting the upper limit of a variable

Symbol denoting the lower limit of a variable

\section{INTRODUCTION}

$\mathbf{T}$ HE increasing penetration of Renewable Energy Sources (RES), especially at electric distribution network level, is increasingly challenging the established network operations

A. Di Giorgio, A. Lanna, A. Pietrabissa and F. Delli Priscoli are with the Department of Computer, Control and Management Engineering, at "Sapienza" University of Rome, Via Ariosto 25, 00185, Rome, Italy, e-mail: \{lanna,digiorgio,pietrabissa,dellipriscoli\}@diag.uniroma1.it.

F. Liberati is with the SMART Engineering Solutions \& Technologies (SMARTEST) Research Center, eCampus University, Via Isimbardi 10, 22060, Novedrate CO, Italy e-mail: francesco.liberati@uniecampus.it.

This work is partially supported by the Sapienza-Ateneo 2014 and 2013 projects no. C26A14YT4 and no. C26A13LYTBX, on optimal ESS control strategies for RES integration in distribution networks. A. Di Giorgio and F. Liberati are co-first authors of this paper. based on the traditional, centralized, and mono-directional paradigm of the grid [1]. In particular, the increasing addition of photovoltaic and wind plants at Low Voltage (LV) and Medium Voltage (MV) levels is making the power profiles at power grid nodes less predictable and controllable, resulting, among the other effects, into the grid possibly working out of the optimal operating points established on the day-ahead basis. As a matter of fact, the traditional operation of today's power systems foresees, generally speaking, a day-ahead planning step, in which the consumption and generation balance is planned, and one or more intraday balancing steps, which put in place corrective actions during the real time operations, in case a mismatch between generation and consumption is observed. The uncertainty and drop in controllability introduced by the massive adoption of RES technology has been putting the research on real time balancing strategies and RES forecasting under the spotlight. The storage technology as well will more and more represent a promising factor for adding flexibility to the grid, as it allows, up to the extent given by the power and energy capacities of the storage devices, to break the need for instantaneous balancing between generation and consumption [2].

In the light of the above, this paper presents a Model Predictive Control (MPC) strategy for controlling an Energy Storage System (ESS) in a power distribution node, with the objective of letting the controlled node power profile track the curve established on the day ahead, while guaranteeing that the RES fluctuations seen at node level are smoothed as much as possible and the ESS state of charge evolution is kept within the saturation limits. In this line of research, ESS control has been the subject of a growing interest, and a number of original contributions have appeared in the relevant literature. An interesting paper based on the same methodology applied here is [3], which presents an MPC framework for smoothing the power output of a wind farm, based on the availability of accurate wind power prediction data. Differently from [3], here we are interested in both power profile smoothing and tracking (thus increasing the effort requested to the ESS). Also, while [3] provides qualitative arguments for proving the stability of the adopted MPC approach, here we propose a rigorous demonstration.

A contribution based on a similar reference scenario is reported in [4], where the objective is that of smoothing the output of a wind farm and tracking a properly generated power reference for the wind farm. The ESS control scheme proposed is simple and foresees that the ESS covers the mismatch between the current power output of the farm and the reference. Contrary to [4], the control strategy presented 
here is expected to guarantee superior performances by solving an optimization problem over a prediction window into the future, thus optimizing system's performances not just in the current time. Another interesting work is [5], which proposes an optimization-based control scheme for an ESS installed in a feeder hosting significant amount of RES. Differently from this paper, the objective of the control scheme is to maximize the economical efficiency of the ESS operations, by properly controlling the charging/discharging frequency and depth. Similarly, [6] presents three ESS control strategies to steer the output of a wind farm (in terms of power or energy) close to the values communicated in the day ahead. The strategies are condition-based and account for, respectively, a "greedy" compensation of the power imbalance, the postcompensation of the energy imbalance and a more refined, two-stages compensation including a forecast of the energy imbalance.

Also storage applications to optimal power flow problems at transmission level have been proposed. Reference [7] addresses the problem from a theoretical perspective, considering a network with a single generator and a single load. Reference [8] instead tackles the problem on a simulation base. Reference [9] provides a proof of concept based on the IEEE 14 bus system. In [10], the integration of ESS and RES is jointly investigated and simulated. Many applied research projects and demonstrators are as well producing efforts to test the feasibility and sustainability of storage integration (see e.g. GRID4EU [11], Energy@ Home [12], [13]).

The distinctive feature of the methodology applied in this work lays in the possibility of optimizing the chosen performance indicators (power smoothing and tracking in this case) not just in the current time but also considering the predicted evolution of the system over a time window in the future. Also, MPC allows to include in the formulation: (i) a model of the system under control (the ESS), (ii) predictions of the variables affecting system's performance (RES output forecasts in this case), (iii) the technical constraints impacting on the control problem. MPC thus results in increased performances, with intrinsic robustness provided by the continuous re-optimization process (see Section III).

A model similar to the one presented here, but applied to a different use case, is found in [14], where a distributed algorithm for MPC of multiple ESSs in a network of residential energy systems is proposed, with the aim of flattening the power demand from the electricity grid, also making proper comparisons with centralized and decentralized approaches. The distributed algorithm is then theoretically investigated in [15], where it is shown to achieve the performance of a large-scale centralized optimization algorithm, having at the same time the advantages coming from distributing the computational effort. Differently from the present work, [15] focuses on the efficient computation of a solution to the openloop optimal control problem solved at each MPC iteration, rather than on the analysis of the closed loop properties of the MPC algorithm. In addition, the formulation proposed in the present work addresses also the requirement of keeping the storage state of charge close to a desired reference, in addition to that of controlling the power profile (this is a

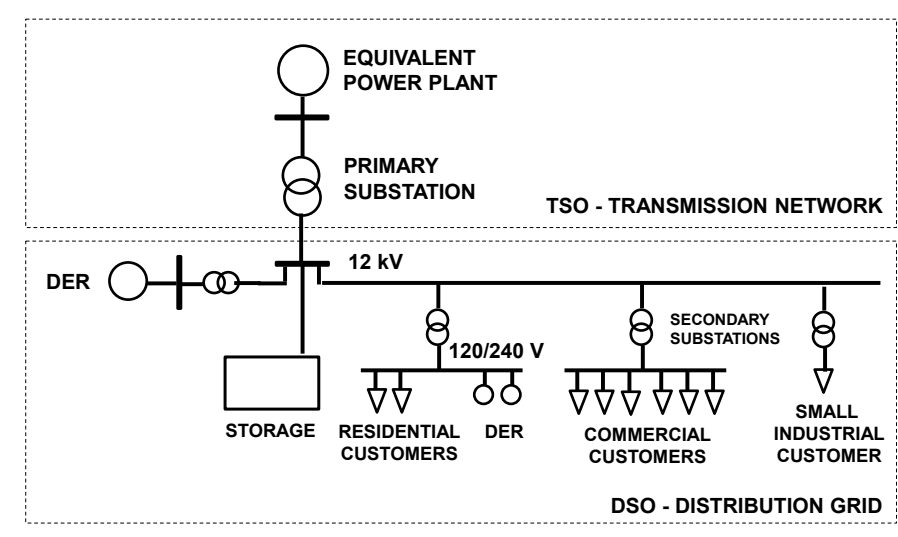

Figure 1. Reference scenario for the proposed ESS integration study.

specific requirement of the tackled use case).

Finally, the research reported in this paper is based on the work in [16], by the same authors. The present work significantly extends the previous work by: (i) modifying the MPC formulation so as to enable the day-ahead power reference tracking functionality; (ii) providing first theoretical results on the stability of the proposed control system in a simplified setting; (iii) performing more extended simulations, by testing the system under real working conditions and in presence of RES forecasting errors.

The remainder of the paper is organized as follows. Section II presents the reference scenario for the proposed ESS integration study. Section III reports the formalization of the related control problem and its theoretical analysis. In Section IV the simulation results are presented. In Section V the conclusions of this work are discussed and future works are outlined.

\section{REFERENCE SCENARIO}

The reference scenario at the basis of this study is depicted in Fig. 1. An HV/MV substation is considered, connected to the transmission network, modeled as an equivalent traditional power plant. The MV feeders host consumption and distributed generation. The reference scenario further considers an electric ESS connected to the MV busbar of the substation, and controlled by the Distribution System Operator (DSO), with the objective of guaranteeing that the net substation power profile is smooth and tracks the profile established on the day ahead by the DSO and the Transmission System Operator (TSO). Such a capability appears fundamental both in view of the possibility of implementing local energy management strategies, but also with reference to the task of extending the traditional power flow calculation to the case of networks with high penetration of renewables. As a matter of fact, standard power flow calculations are based on the assumption that substation nodes can be associated with highly predictable (consumption) profiles. This assumption is challenged by the increasing penetration of RES, but can be restored precisely by the proposed control scheme.

The considered scenario can help addressing also similar problems, as the one of controlling a stand-alone local power system operated in islanding mode, where a large load is fed by a real power station with the support of RES and ESS. Finally, the proposed MPC control algorithm can be hosted 


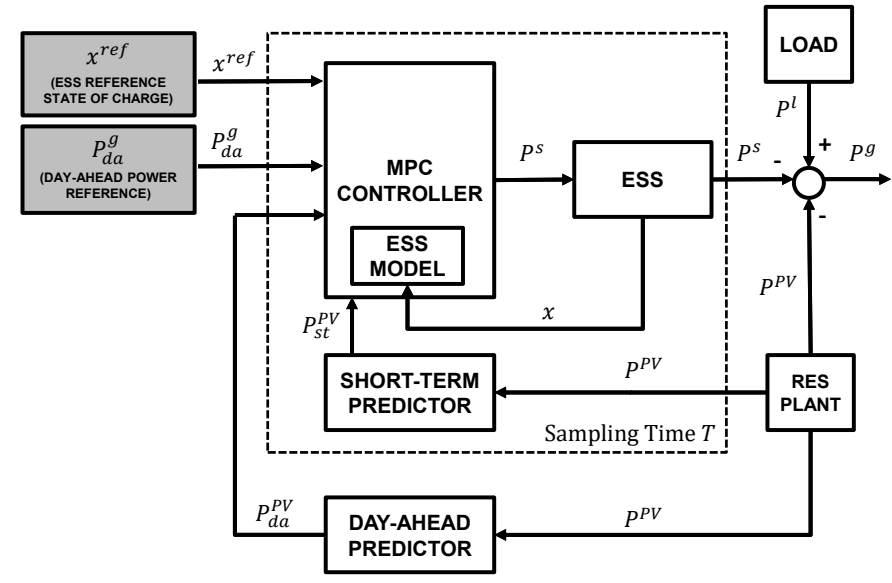

Figure 2. Block diagram of the proposed control scheme.

either at the level of the DSO SCADA control centre, or locally at the ESS level, with field data retrieved, respectively, through a telecommunication infrastructure or through the DSO electric infrastructure (e.g. through Power Line Communications (PLC) [17]).

\section{ESS CONTROL SCHEME}

This section details the proposed ESS control strategy, starting from the control system modeling. Figure 2 reports the block diagram of the controlled ESS. Two RES output predictors are considered in the control scheme. A first predictor operates on a day-ahead basis, and the resulting forecast is used to estimate the net node consumption to be traded on the day-ahead markets. A second short-term predictor provides the MPC controller with refined RES forecasts for increased control performance, as discussed next. Two reference inputs are considered (gray blocks in the figure): (i) $x^{r e f}$, a reference value for the storage state of charge operations, and (ii) $P_{d a}^{g}$, the net node consumption planned on the day-ahead. The remaining inputs to the controller are: (i) the day ahead RES forecast $P_{d a}^{p v}$, (ii) the short term RES forecast $P_{s t}^{p v}$ (potentially updated at each control iteration), (iii) the measured ESS state of charge. The output of the controller is the control profile for the ESS. The control objective is to control the node power exchange as to track in time the day-ahead reference $P_{d a}^{g}$, while keeping the ESS state of charge evolution as much as possible close to the reference value $x^{r e f}$, in order to guarantee controllability margins for intervention following a grid contingency.

The control problem is tackled via discrete-time MPC [18]. According to this strategy, at each sampling time the current ESS control is found by solving on-line a finite horizon openloop optimal control problem, using the current state of the plant (i.e. the current measure of the ESS state of charge) as the initial state of the optimization problem, then achieving a closed-loop control system. The first sample of the computed optimal control sequence is applied to the plant; the remaining part of the control sequence is discarded. The mentioned openloop optimal control problem encodes the control objectives in the target function, and includes proper constraints to account for the limitations of the devices involved.
The detailed mathematical formulation of the open-loop optimal control problem solved at the generic time $k$ is detailed in the next section. In the following, $N \in \mathbb{N}$ denotes the MPC control horizon and $T \in \mathbb{R}$ the discretization step.

\section{A. Objective Function}

The selected target function aims at establishing a trade-off between the need of tracking the net node power flow planned the day-ahead, and that of avoiding large excursions in the storage state of charge. The target function is denoted by $V_{N}$, where $N$ reminds that the problem is solved on an horizon of $N$ time steps ahead of the current time $k$.

$$
\begin{aligned}
V_{N}(k)=\sum_{i=1}^{N} & \left\{\alpha(i)\left[P_{d a}^{g}(k+i-1)-P_{s t}^{g}(k+i-1)\right]^{2}\right. \\
& \left.+\beta(i)\left[x(k+i-1)-x^{\mathrm{ref}}\right]^{2}\right\}
\end{aligned}
$$

The first term of the objective function accounts for the power tracking error along the control horizon, that is, the mismatch between the net reference active power exchange $P_{d a}^{g}$ established in the day ahead (based on day-ahead forecasts of the RES power), and a more accurate short-term estimate $P_{s t}^{g}$ of the same power exchange. Parameter $\alpha(i)$ weights the power tracking error along the control horizon. The second term of the target function is introduced to limit the excursion of the ESS state of charge with respect to its defined reference value (parameter $\beta$ has a similar meaning as $\alpha$ ). Parameters $\alpha$ and $\beta$ are non-negative.

\section{B. Constraints}

The constraints included in the generic MPC iteration are detailed hereafter. First of all, an ESS system dynamics and proper power balance equations have to be included in order to explicitly write the two objective function's terms in function of the problem control variables (given by the ESS power setpoint $P^{s}$ along the control horizon). Regarding the ESS model, the following linear, first-order dynamics is introduced

$$
\begin{aligned}
& x(i+1)=x(i)-T P^{s}(i) \quad \forall i \in[k, k+N-1] \\
& x(k)=x_{k}
\end{aligned}
$$

where $x_{k}$ denotes the feedback of the ESS state of charge acquired at $k$. The following power balance equation can be used to rewrite the first term of the objective function (variable $\left.P_{s t}^{g}\right)$ as a function of the ESS control variable

$$
P^{g}(i)+P^{p v}(i)+P^{s}(i)=P^{l}(i) \quad \forall i \in[k, k+N-1]
$$

From the above balance equation the short term forecast $P_{s t}^{g}$ of the power exchange at substation level easily follows

$$
P_{s t}^{g}(i)+P_{s t}^{p v}(i)+P^{s}(i)=P^{l}(i) \quad \forall i \in[k, k+N-1]
$$

On the day-ahead basis instead, $P_{d a}^{g}$ is computed not taking into account any contribution from the ESS.

$$
P_{d a}^{g}(i)+P_{d a}^{p v}(i)=P^{l}(i) \quad \forall i \in[k, k+N-1]
$$

Finally, proper box constraints are introduced in order to keep physical variables within their acceptable operating ranges. 
The restrictions to be considered here are on the active power exchange and the ESS state of charge, limited as follows

$$
\begin{aligned}
\check{P}_{s t}^{g} & \leq P_{s t}^{g}(i) \leq \hat{P}_{s t}^{g} \\
\check{P}^{s} & \leq P^{s}(i) \leq \hat{P}^{s} \quad \forall i \in[k, k+N-1] \\
\check{x} & \leq x(i+1) \leq \hat{x}
\end{aligned}
$$

where symbols $:$ and $\hat{r}$ denote lower and upper limits. The above constraints ensure that the computed ESS control always results in feasible ESS state of charge and power control trajectories.

\section{MPC Iteration}

The generic MPC iteration can be stated as follows.

Problem 1 (ESS MPC iteration at generic time k). For the given power substation with known demand $P^{l}$, known RES day-ahead forecast $P_{d a}^{p v}$, known RES short-term forecast $P_{s t}^{p v}(i), i \in[k, k+N-1]$ and reference ESS state of charge $x^{r e f}$, find the optimal ESS control sequence $P^{s}(i), i \in[k, k+$ $N-1]$ which minimizes (1) subject to the ESS dynamics (2) - with initial ESS state of charge known from feedback -, the balance constraints (4)-(5) and the bounds (6).

The above problem is a quadratic constrained programming problem. By simple manipulations, it can be put in the following form

$$
\begin{gathered}
\min _{\mathbf{u}(k)}\left\{V(k)=\frac{1}{2} \mathbf{u}(k)^{T} \mathbf{H}(k) \mathbf{u}(k)+\mathbf{f}^{T}(k) \mathbf{u}(k)\right\} \\
\mathbf{A}_{\text {ineq }}(k) \mathbf{u}(k) \leq \mathbf{B}_{\text {ineq }}(k) \\
\mathbf{A}_{\text {eq }}(k) \mathbf{u}(k)=\mathbf{B}_{\text {eq }}(k) \\
\mathbf{u}_{\text {min }} \leq \mathbf{u}(k) \leq \mathbf{u}_{\text {max }}
\end{gathered}
$$

where the column vector $\mathbf{u}(k)$ is the optimization vector given by the collection of control variables $P^{s}$ over the control horizon $\left(\mathbf{u}(k)=\left[P^{s}(k), P^{s}(k+1), \ldots, P^{s}(k+N-1)\right]^{T} \in \mathbb{R}^{N}\right)$. In detail, (7) can be derived by re-writing (1) in matrix form, by substituting $P_{s t}^{g}(t)$ and $P_{d a}^{g}(t)$ according to the equations (4) and (5), and after having found the explicit solutions of (2) (i.e. after writing $x(k+i-1), i \in[1, N]$ in (1) as function of the current state $x(k)$ and of the control sequence $\left.\left\{P^{s}(k), P^{s}(k+1), \ldots, P^{s}(k+i-1)\right\}\right)$. In a similar way, constraints (3)-(5) can be put in the form of the equality constraints (9), while the set of bounds (6) can be put in the form of the inequality constraints (8) and the box constraints (10). $\mathbf{H}, \mathbf{f}, \mathbf{A}_{\text {ineq }}, \mathbf{B}_{\text {ineq }}, \mathbf{A}_{e q}, \mathbf{B}_{\text {eq }}$ are coefficient matrices and vectors of proper dimensions and are updated at each iteration.

The above formulation is the one used for implementation purpose. In this work, the algorithm used to solve Problem 1 is the barrier method [19] as implemented in Gurobi v. 6.0.4.

\section{Stability Analysis}

This section discusses the stability properties of the proposed MPC control scheme. To this end, the MPC optimization problem is rewritten as follows (time indices are omitted whenever possible). By considering (4) and (5), the term $\left(P_{d a}^{g}-P_{s t}^{g}\right)$ in (1) is written as $\left(u+e^{p v}\right)$, where $e^{p v}=P_{s t}^{p v}-P_{d a}^{p v}$ and $u=P^{s}$ denotes the control input to the ESS. Also, by the suitable change of coordinates $\tilde{x}:=x-x^{r e f}$, the term $\left(x-x^{r e f}\right)$ is simplified as $\tilde{x}$. Let us further concisely denote by $\tilde{x}(i+1)=f(\tilde{x}(i), u(i))$ the difference equation (2), written in the new coordinates. The objective function of the MPC scheme at the generic time $k$, written as a function $V_{N}$ of the ESS state of charge $\tilde{x}(k)$ and of the control sequence $\mathbf{u}(k)=\{u(k), u(k+1), \ldots, u(k+N-1)\}$, can be therefore written as $V_{N}(\tilde{x}, \mathbf{u}, k)=$ $\sum_{i=1}^{N}\left\{\alpha(i)\left[u(k+i-1)+e^{p v}(k+i-1)\right]^{2}+\beta(i) \tilde{x}(k+i-1)^{2}\right\}$. The analysis is restricted in the following to the case of zero forecasting error $\left(e^{p v} \equiv 0\right)$. Under that assumption, the problem is time-invariant, in the sense that $V_{N}(\tilde{x}, \mathbf{u}, k)=V_{N}(\tilde{x}, \mathbf{u}, 1)$, that is, the open-loop problem defined at time $k$ starting from initial state $\tilde{x}$ is equal to the problem defined at 1 starting from state $\tilde{x}$. Therefore, it is possible to write

$$
\begin{array}{ll}
\min _{\mathbf{u}}\left\{V_{N}(\tilde{x}, \mathbf{u})\right. & =\sum_{i=1}^{N}\left\{\alpha(i) u(i)^{2}+\beta(i) \tilde{x}(i)^{2}\right\} \\
& \left.=\sum_{i=1}^{N} l(\tilde{x}(i), u(i), i)\right\} \\
\text { s.t. } & \tilde{x}(i) \in \mathbb{X}, u(i) \in \mathbb{U}, \quad i=1, \ldots, N \\
& \tilde{x}(i+1)=f(\tilde{x}(i), u(i)), \quad i=1, \ldots, N-1 \\
& \tilde{x}(1)=\tilde{x},
\end{array}
$$

where we have concisely indicated with $l$ the stage cost, and the compact sets $\mathbb{X}$ and $\mathbb{U}$ are defined based on the box constraints in (6). $\tilde{x}$ is the initial state of the system (in the new coordinates) and can be regarded as a constant when solving the open loop control problem (note however that the state trajectory evolves over time as a result of the application of the first sample of the found optimal control sequence). The following notation is further assumed. Let $\mathbf{u}^{0}(\tilde{x}) \in \mathbb{R}^{N}$ denote the optimal solution of (11)-(12) (notice that the optimization problem always admits an optimal solution, since all the involved functions are continuous, the problem is convex and defined over a compact set). Let $u^{0}(\tilde{x}, i) \in \mathbb{R}$ denote the i-th sample of $\mathbf{u}^{0}(\tilde{x})$ (i.e. $\left.\mathbf{u}^{0}(\tilde{x})=\left\{u^{0}(\tilde{x}, 1), u^{0}(\tilde{x}, 2), \ldots, u^{0}(\tilde{x}, N)\right\}\right)$. Let $V_{N}^{0}(\tilde{x}) \in \mathbb{R}$ denote the value of the target function attained in the minimizer $\mathbf{u}^{0}(\tilde{x})$, that is $V_{N}^{0}(\tilde{x}):=V_{N}\left(\tilde{x}, \mathbf{u}^{0}(\tilde{x})\right)$. Let $\tilde{x}^{0}(\tilde{x}, i)$ denote the state reached when the optimal sequence $\left\{u^{0}(\tilde{x}, 1), u^{0}(\tilde{x}, 2), \ldots, u^{0}(\tilde{x}, i-1)\right\}$ is applied to the system starting from the initial state $\tilde{x}$. The MPC iteration implicitly defines a feedback law $\mathrm{K}$ such that $\mathrm{K}(\tilde{x}):=u^{0}(\tilde{x}, 1)$ (in fact, recall that the MPC iteration consists in solving an openloop optimal control problem and then applying to the system the first sample of the optimal control sequence). The aim of this section is to assess the stability properties of the $M P C$ controlled system $\tilde{x}(k+1)=f(\tilde{x}(k), \mathrm{K}(\tilde{x}(k)))$, under the case $e^{p v} \equiv 0$. It is shown next that $\tilde{x}=0$ (i.e. $x=x^{r e f}$ ) is a globally asymptotically stable equilibrium point for the controlled system (that is, $f(\tilde{x}(k), \mathrm{K}(\tilde{x}(k))) \rightarrow 0$ as $k \rightarrow \infty$, for every value of the initial state). The following lemma is instrumental to the main demonstration.

Lemma 1. Assuming $e^{p v} \equiv 0$, the inequality $l\left(f\left(\tilde{x}^{0}(\tilde{x}, N), u^{0}(\tilde{x}, N)\right), 0, N\right)-l(\tilde{x}, \mathrm{~K}(\tilde{x}), 1) \leq-\eta(\tilde{x})$ 
holds for the system at study, with $\eta$ a continuous function strictly positive definite (i.e. $\eta(0)=0$ and $\eta(\cdot)>0$ elsewhere).

Proof. First of all, $u^{0}(\tilde{x}, N)=0 \quad$ and $f\left(\tilde{x}^{0}(\tilde{x}, N), u^{0}(\tilde{x}, N)\right)=\tilde{x}^{0}(\tilde{x}, N)$ for the particular system at study. $u^{0}(\tilde{x}, N)=0$ results from the fact that no terms in the objective function refer to the state $\tilde{x}(N+1)$ at the end of the control window, and thus the choice $u^{0}(\tilde{x}, N)=0$ is both feasible and optimal (it is associated to the least cost, since the target function is quadratic). $f\left(\tilde{x}^{0}(\tilde{x}, N), 0\right)=\tilde{x}^{0}(\tilde{x}, N)$ results from the fact that for the system in question the dynamics $\tilde{x}(k+1)=f(\tilde{x}(k), u(k))$ is linear and given by $\tilde{x}(k+1)=\tilde{x}(k)-T u(k)$ (see (2)).

Given the structure of $l$ for the problem in question (see (11)), the lemma is proven if it is shown that $\left|\tilde{x}^{0}(\tilde{x}, N)\right| \leq$ $c|\tilde{x}| \forall \tilde{x} \neq 0$, with $c<1$ a positive constant. In fact, direct substitution shows that in this case the lemma holds true with $\eta=\left[\beta(1)-\beta(N) c^{2}\right] \tilde{x}^{2}$ and provided $\beta$ satisfies the additional sufficient condition $\left[\beta(1)-\beta(N) c^{2}\right] \geq 0$.

Let us then consider the case $\tilde{x}>0$, that is, $x \geq$ $x^{\text {ref }}$ (the case in which $\tilde{x}<0$ can be treated similarly). For $\tilde{x}>0, u^{0}(\tilde{x}, i) \geq 0 \forall i$, and hence the sequence $\tilde{x}^{0}(\tilde{x}, \cdot)$ is non increasing ${ }^{1}$. Similarly, it can be shown that $\tilde{x}^{0}(\tilde{x}, i) \geq 0 \forall i$. By the dynamic programming principle ${ }^{2}$, $u^{0}(\tilde{x}, N-1)=\operatorname{argmin}_{u}\left\{\beta(N-1) \tilde{x}^{0}(\tilde{x}, N-1)^{2}+\alpha(N-\right.$ 1) $\left.u^{2}+\beta(N)\left[\tilde{x}^{0}(\tilde{x}, N-1)-T u\right]^{2}\right\}=\operatorname{argmin}_{u}\{[\alpha(N-1)+$ $\left.\left.\beta(N) T^{2}\right] u^{2}-2 \beta(N) T \tilde{x}^{0}(\tilde{x}, N-1) u\right\}$, subject to the constraint $u \leq \hat{u}$, which yields $u^{0}(\tilde{x}, N-1)=\min \left\{\beta(N) T \tilde{x}^{0}(x, N-\right.$ 1) $\left./\left[\alpha(N-1)+\beta(N) T^{2}\right], \hat{u}\right\}$, with $\hat{u}=\hat{P}^{s}$. From the dynamic equation $\tilde{x}^{0}(\tilde{x}, N)=\tilde{x}^{0}(\tilde{x}, N-1)-T u^{0}(\tilde{x}, N-1)$ it can be checked that both the possible values for $u^{0}(\tilde{x}, N-1)$ result in a feasible state $\tilde{x}^{0}(\tilde{x}, N)$ (in particular, $\tilde{x}^{0}(\tilde{x}, N-1) \geq$ $\left.\tilde{x}^{0}(\tilde{x}, N) \geq 0\right)$. Then, when $u^{0}(\tilde{x}, N-1)=\beta(N) T \tilde{x}^{0}(x, N-$ 1) $/\left[\alpha(N-1)+\beta(N) T^{2}\right]$, direct calculation shows that $\tilde{x}^{0}(\tilde{x}, N) / \tilde{x}^{0}(\tilde{x}, N-1)=\alpha(N-1) /\left[\alpha(N-1)+\beta(N) T^{2}\right] \leq 1$. If instead $u^{0}(\tilde{x}, N-1)=\hat{u}$ (i.e., when the control saturation condition $\beta(N) T \tilde{x}^{0}(x, N-1) /\left[\alpha(N-1)+\beta(N) T^{2}\right] \geq \hat{u}$ holds), we have $\tilde{x}^{0}(\tilde{x}, N) / \tilde{x}^{0}(\tilde{x}, N-1)=1-T \hat{u} / \tilde{x}^{0}(\tilde{x}, N-$ $1) \leq 1$, since, from the saturation condition it is $T \hat{u} / \tilde{x}^{0}(\tilde{x}, N-$ $1) \leq \beta(N) T^{2} /\left[\alpha(N-1)+\beta(N) T^{2}\right] \leq 1$. Hence, it can be concluded that (recall that the state trajectory is positive and non-increasing) $\tilde{x}^{0}(\tilde{x}, N) / \tilde{x} \leq \tilde{x}^{0}(\tilde{x}, N) / \tilde{x}^{0}(\tilde{x}, N-1) \leq$ $\max \left\{\alpha(N-1) /\left[\alpha(N-1)+\beta(N) T^{2}\right], 1-T \hat{u} / \tilde{x}^{0}(\tilde{x}, N-\right.$ $1)\} \leq \max \left\{\alpha(N-1) /\left[\alpha(N-1)+\beta(N) T^{2}\right], 1-T \hat{u} / \hat{\tilde{x}}\right\}$. In conclusion, we have found that $\tilde{x}^{0}(\tilde{x}, N) / \tilde{x} \leq \max \{\alpha(N-$ 1) $\left./\left[\alpha(N-1)+\beta(N) T^{2}\right], 1-T \hat{u} / \hat{\tilde{x}}\right\} \leq 1$, and therefore the lemma is proved with $c=\max \{\alpha(N-1) /[\alpha(N-1)+$ $\left.\left.\beta(N) T^{2}\right], 1-T \hat{u} / \hat{\tilde{x}}\right\}$ and $\eta=\left[\beta(1)-\beta(N) c^{2}\right] \tilde{x}^{2}$.

\footnotetext{
${ }^{1}$ In fact, if there were some $i$ in the optimal control sequence for which $u^{0}(\tilde{x}, i) \leq 0$, then the sequence with $u^{0}(\tilde{x}, i)=0$ for the same values of $i$ would be feasible and with a smaller cost associated (as it can be seen considering the structure of $l$ in (11) and the dynamics of the system (2)).

${ }^{2}$ Suppose we know the optimal sequence $\left\{u^{0}(\tilde{x}, 1), u^{0}(\tilde{x}, 2), \ldots, u^{0}(\tilde{x}, N-2)\right\}$, the remaining optimal control samples $u^{0}(\tilde{x}, N-1)$ and $u^{0}(\tilde{x}, N)$ are the ones yielding the value $V_{2}^{0}\left(\tilde{x}^{0}(\tilde{x}, N-1)\right)$. When solving the "truncated" optimization problem $\left\{\min _{\mathbf{u}} V_{2}\left(\tilde{x}^{0}(\tilde{x}, N-1), \mathbf{u}\right)\right.$ subject to $\left.(12)\right\}, \tilde{x}^{0}(\tilde{x}, N-1)$ can be treated as a constant, and the fact that $u^{0}(\tilde{x}, N)=0$ can be exploited. Notice that from (2) we can write $\tilde{x}^{0}(\tilde{x}, N)=\tilde{x}^{0}(\tilde{x}, N-1)-T u$.
}

Theorem 1. Assuming $e^{p v} \equiv 0, \tilde{x}=0$ (i.e. $x=x^{r e f}$ ) is a globally (i.e., for all initial conditions in $\mathbb{X}$ ) asymptotically stable equilibrium point for the controlled system $\tilde{x}(k+1)=$ $f(\tilde{x}(k), \mathrm{K}(\tilde{x}(k)))$.

Proof. The state $\tilde{x}=0$ is an equilibrium point for the controlled system, since $\mathrm{K}(0)=0$ and $f(0,0)=0$. Stability can be proven via the Lyapunov direct method (see e.g. [18]). At time $k+1$, the control sequence $\tilde{\mathbf{u}}(k+$ $1)=\left\{u^{0}(\tilde{x}, 2), u^{0}(\tilde{x}, 3), \ldots, u^{0}(\tilde{x}, N), 0\right\}$ is feasible for the MPC iteration (and hence the associated value of the target function is not lower than the optimal one). It follows that $V_{N}^{0}(f(\tilde{x}, \mathrm{~K}(\tilde{x}))) \leq V_{N}(f(\tilde{x}, \mathrm{~K}(\tilde{x})), \tilde{\mathbf{u}})=V_{N}^{0}(\tilde{x})-$ $l(\tilde{x}, \mathrm{~K}(x), 1)+l\left(f\left(\tilde{x}^{0}(\tilde{x}, N), u^{0}(\tilde{x}, N)\right), 0, N\right)$. By the lemma above, $V_{N}^{0}(f(\tilde{x}, \mathrm{~K}(\tilde{x})))-V_{N}^{0}(\tilde{x}) \leq-\eta(\tilde{x})$, with $\eta$ a continuous function strictly positive definite (i.e. $\eta(0)=0$ and $\eta(\cdot)>0$ elsewhere). The sequence $V_{N}^{0}$ is thus non-increasing, and therefore convergent ( $V$ is lower bounded by zero). Hence it follows that $\eta(\tilde{x}) \leq V_{N}^{0}(\tilde{x})-V_{N}^{0}(f(\tilde{x}, \mathrm{~K}(\tilde{x}))) \rightarrow 0$ as $k \rightarrow \infty$, which proves the asymptotic global stability of the system, since, due to the positive definiteness of $\eta, \eta \rightarrow 0$ implies that $x \rightarrow 0$.

The case $e^{p v} \neq 0$ appears inherently more complex to analyze, as it implies solving a state regulation problem and a control tracking problem, something which is not typically found in MPC theoretical studies. For this reason, the case $e^{p v} \neq 0$ is demanded to future research and is addressed in this work on a simulation basis.

\section{RESUlts}

The proposed approach has been simulated in a specific case study, which considers an HV/MV substation equipped with two transformers, each one of 60 MVA. The substation is connected to the transmission network, modeled as an equivalent generator characterized by unlimited active power capacity $P^{g}$. On the MV side, the ESS is assumed directly connected to the MV busbar, and has $12 \mathrm{MWh}$ of storage maximum capacity and $P^{s}= \pm 6 \mathrm{MW}$ of nominal power. The lower ESS state of charge limit is set to $\check{x}=0 \mathrm{MWh}$. The upper limit is set to $\hat{x}=12 \mathrm{MWh}$. An equivalent PV system with $P^{p v}=10 \mathrm{MW}$ peak power models the set of RES plants in the MV network. Finally, demand $P^{l}$ is modeled as an aggregated power load. Regarding the controller parameters, the considered sampling period $T$ has been set to 5 minutes and the control horizon $N$ to an equivalent of 2 hours, unless differently indicated. In all the simulations, the reference state of charge of the ESS is set to half of the maximum ESS energy capacity $\left(x^{r e f}=6 \mathrm{MWh}\right)$, for all the simulation time, in order to provide equal energy injection and absorption margins. In practice, the reference storage state of charge value is decided by the system operator, and all the values between the minimum and the maximum capacity can be chosen as reference values.

Simulations have been performed using a Macbook 5.2, Intel Core 2 Duo, $2.13 \mathrm{GHz}$, 5GB RAM computer, running Apple OSX v. 10.11.1. The control framework has been built in Matlab R2012a, and Gurobi v. 6.0.4 has been used to solve 
the MPC iterations. The average solving time was $0.059 \mathrm{~s}$, including the pre-processing and post-elaboration phases.

Three simulations have been performed for the assessment of the control system performance at steady state and in response to fluctuations in the power production from RES. The first two simulations are intended to provide a numerical proof of concept of the system; the third simulation refers to the system working in real conditions, with real input data.

For each simulated scenario, performances vary depending on the type of the information provided to the controller regarding the RES prediction $P_{s t}^{p v}$. Three cases are distinguished in the following:

- Case 1: No short term RES predictions are available to the controller. The controller works based uniquely on the day-ahead prediction of the RES output (that is, $P_{s t}^{g}$ in (1) is computed assuming $P_{s t}^{p v}=P_{d a}^{p v}$ in (4)). Results from this configuration provide the baseline against which the improvements brought by the proposed ESS control system can be evaluated. As a matter of fact, notice from (1), (4) and (5) that, if $P_{s t}^{p v}=P_{d a}^{p v}$, when the state of charge is at the reference value, the control output is identically zero, thus coinciding with the uncontrolled case in which the storage is out of the control problem and the node flow is governed uniquely by the balance equation (3). For that reason, with a little abuse of terminology, this case is referred to in the following also as the uncontrolled case.

- Case 2: Perfect short term RES predictions are available to the controller $\left(P_{s t}^{p v}=P^{p v}\right)$. This ideal configuration results in the best possible performance achievable by the system.

- Case 3: The controller works based on short term RES predictions affected by a prediction error $\left(P_{s t}^{p v} \neq P^{p v}\right)$. The prediction error has been chosen in line with that of the current state of the art forecasting systems available in literature [20], [21]. This configuration allows to evaluate the expected performance of the system in a realistic setting. Since the simulation outcome depends in this case on the particular realization of the short term RES prediction, a Monte Carlo approach has been adopted by performing 1000 experiments for each simulation, each experiment being characterized by a different realization of the short term RES forecasting.

For the sake of clearness, we report in the following the additional notation used throughout the simulation section:

- $P^{g, 1}$ is the MPC-controlled profile at the substation achieved when no short term RES predictions are available to the controller (Case 1 above).

- $P^{g, 2}$ is the MPC-controlled profile at the substation achieved when ideal short-term RES forecasting is assumed (Case 2 above).

- $P^{g, 3}$ is the MPC-controlled profile at the substation achieved when short-term predictions affected by errors are available to the controller (Case 3 above).

- In a similar way, $P^{s, 1}, P^{s, 2}$ and $P^{s, 3}$ represent the storage controlled power achieved when, respectively, only day-ahead RES predictions (i.e. Case 1), ideal short term
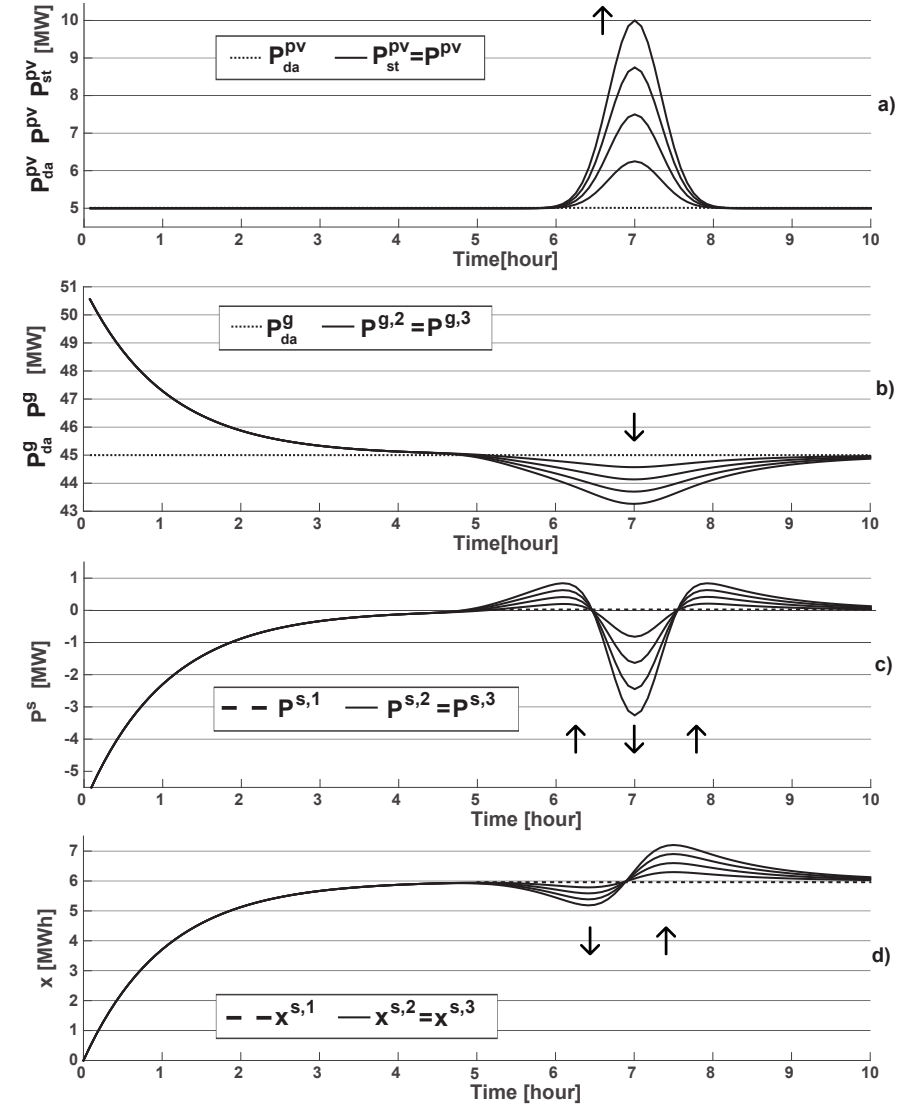

Figure 3. Simulation 1a: RES generation (a), traditional power plant (b), storage active power flow (c) and storage state of charge (d) for increasing values of the forecasting error.

predictions (i.e Case 2) or short term predictions affected by errors (i.e. Case 3 ) are available to the controller.

- Finally, $x^{s, 1}, x^{s, 2}$ and $x^{s, 3}$ have similar meaning referred to the storage state of charge. $x_{\text {ref }}$ denotes the reference storage state of charge.

In conclusion, please note that $P_{s t}^{g}, P_{d a}^{g}$ are predictions of the node power flow (they derive from (4) and (5), respectively). $P^{g, 1}, P^{g, 2}$ and $P^{g, 3}$ instead are actual node flows, derived from (3) considering the particular storage control action $P^{s}$ resulting from MPC. The control action varies depending on the information provided to the controller about the RES output (respectively, only day ahead forecast, perfect forecast and forecast affected by error).

\section{A. Simulation 1: fluctuation of the RES generation}

The simulation carried out in the following is intended to illustrate how the control system reacts to mismatches between the day ahead RES forecast and the short term one. Ideal short term RES forecasting is assumed here (the short term forecast coincides with the real RES output, i.e. $P_{s t}^{p v}=P^{p v}$ ). In this simulation, $P^{l}$ is flat at $50 \mathrm{MW}$. The day ahead prediction $P_{d a}^{p v}$ for the RES output is flat as well, at $5 \mathrm{MW}$. Prediction mismatches are modeled as an additive Gaussian curve centered at hour $7^{, 00}$, with variance equal to 4 and varying amplitude (Fig. 3a). The choice of a Gaussian curve here is motivated only by the simplicity it offers to simulate a peak in RES prediction mismatch, with tunable amplitude and duration (the 

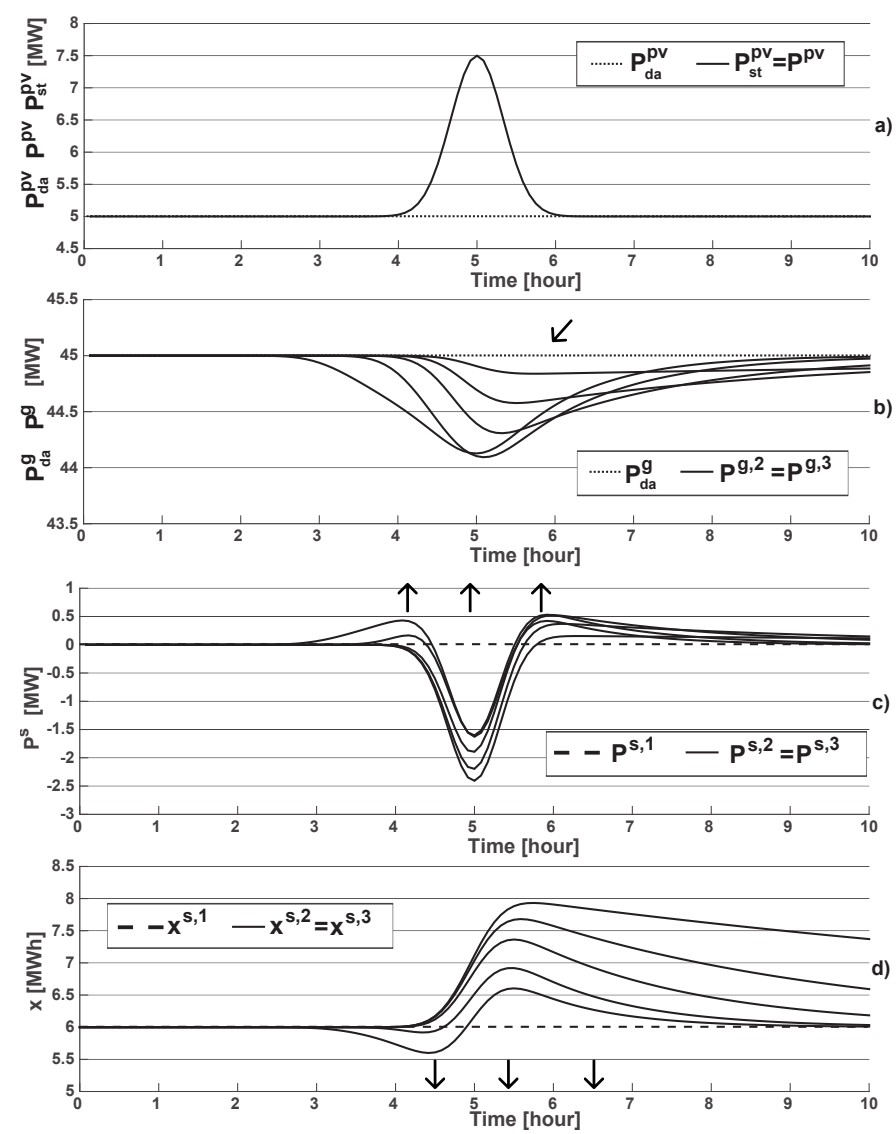

Figure 4. Simulation 1b: RES generation (a), traditional power plant power flow (b), storage active power flow (c) and storage state of charge (d) for increasing values of the control horizon $N$.

third simulation detailed in the following will test the system under realistic RES generation profiles and prediction errors). In this simulation, the system starts from a fully discharged ESS condition (see Fig.3d). The stability of the controlled system in absence of forecast mismatches (i.e. before $6^{, 00}$ ) is confirmed by the simulation (see Fig. 3d), which shows that the ESS state of charge converges towards the reference state of charge. As soon as the forecast mismatch enters the control horizon, the storage starts releasing energy (see Fig. $3 d$ ). Then, as the maximum of the RES generation peak is reached by the control window, the storage comes back to accumulate energy, reaching the minimum in correspondence of the maximum of RES generation (Fig. 3a, hour $7^{, 00}$ ). After that, the ESS state of charge continues to increase for some time, before finally recovering the steady-state value, ready for new interventions. It is to remark that, in absence of the controlled ESS, RES fluctuations (Fig. 3a) would be covered by the traditional power plant. The proposed strategy instead guarantees significant smoothing of $P^{g}$ fluctuations (Fig. 3b).

A second simulation is carried out next to evaluate the effect of varying the control horizon $N$. For convenience of results charting, the initial ESS state of charge is set to the reference value. The profile of the forecasting mismatch assumed is depicted in Fig. 4a. All the remaining simulation parameters and values are kept unchanged. The control horizon is varied between 5 minutes to two hours ( 5 minutes, 15 minutes, 30 minutes, one hour and two hours). Results show that small
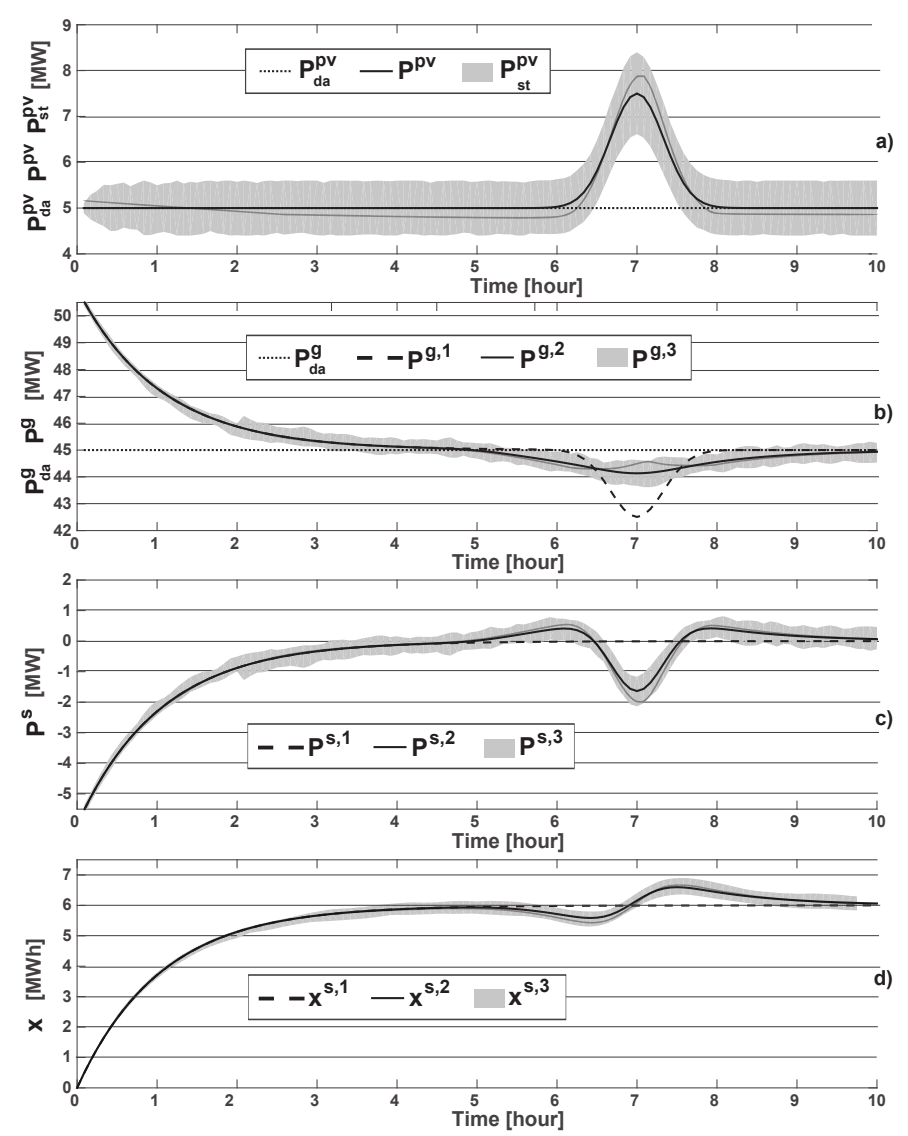

Figure 5. Simulation 2: RES generation (a), traditional power plant power flow (b), storage active power flow (c) and storage state of charge (d).

control horizons are associated with large ESS control efforts, resulting in good peak shaving performances (Fig. 4b), large distance between the ESS state trajectory and the reference value, and long ESS state settling times (Fig. 4d). The arrows in the figure indicate increasing values of the control horizon.

\section{B. Simulation 2: performance under RES forecasting errors}

The second simulation aims at evaluating the impact of short term prediction errors (while in the previous simulation perfect short term forecasting was assumed). The simulation setup is the same as the previous simulation, namely, flat active power demand $P^{l}=50 \mathrm{MW}$ and RES power generation characterized by a power base value of $P^{p v}=5 \mathrm{MW}$ with superimposed a Gaussian profile. It is assumed that the day ahead a flat RES output is predicted $\left(P_{d a}^{p v}=5 \mathrm{MW}\right)$. Results are presented in Fig. 5. Figure 5.a reports the real RES output $P^{p v}$, the flat day ahead RES forecast $P_{d a}^{p v}$, the 1000 different realizations of the RES short term forecast $P_{s t}^{p v}$ (gray lines forming the gray band) and, finally, one particular realization of the RES short term forecast (dark gray line). Figure 5.b shows that, when the MPC controller works based on the day ahead RES prediction, all the effort in matching the RES fluctuation is demanded to the grid, with consequent large fluctuation of the power exchanged at node level (see $P^{g, 1}$ ). On the other hand, ideal short term forecasting results in the best possible flattening performances, as the RES fluctuation is smoothed by the storage action, with mitigated impact on the 
node power exchange (see $P^{g, 2}$ in Fig. 5.b). The same good performance is observed in the 1000 experiments considering the availability of real short term predictions affected by errors (see the gray band $P^{g, 3}$ in Fig. 5.b), which significantly outperform the uncontrolled case (see $P^{g, 1}$ in the figure). Figure $5 \mathrm{c}$-d report, respectively, the ESS control and state of charge evolution.

Finally, the next simulations presented in Fig. 6 show how the performance of the controlled system changes when acting on the weights $\alpha(i)$ and $\beta(i)$ in the objective function (1), and on the length of the control horizon. To this end, we take $\alpha(i)=\alpha$ and $\beta(i)=\beta=(1-\alpha) \forall i$, with $\alpha \in[0,1]$. Five batches of experiments are performed, one for each value of the control horizon length, chosen in the set $[1,3,6,12,24]$. Results are shown in Fig. 6a-e, for increasing values of the control horizon length. For each batch of experiments, the same simulation as the one shown previously in this section is run with the chosen length of the control horizon and with parameter $\alpha$ varying from 0 to 1 , with steps of 0.05 . Again, results are presented for the uncontrolled case (no short term prediction available), for the case with ideal short term prediction and the one with real short term predictions (1000 simulations). In each of the sub-figures, the $\mathrm{x}$-axis reports the storage tracking error $\left(\sum_{i}\left(x(i)-x^{\mathrm{ref}}\right)^{2}\right)$, while the $\mathrm{y}$-axis reports the power tracking error $\left(\sum_{i}\left(P_{d a}^{g}(i)-P_{s t}^{g}(i)\right)^{2}\right)$. It is seen from the simulations that low values of the control horizon length result in increased power tracking, at the expense of the storage state of charge tracking. The opposite happens when $N$ increases. A balanced configuration, which allows to fine control the storage/power reference tracking tradeoff with uniform sensitivity along the tradeoff curve results from value $N=3$.

\section{Simulation 3: real demand and RES generation profiles}

In the third simulation, real bus demand (Fig. 7a) and RES output profiles (Fig. 7b) have been considered. Figure 7 shows the results in the three cases (uncontrolled, perfect RES forecasting and real short term forecasting). Results show that the proposed controller effectively smoothens the power curve in presence of RES fluctuations, always keeping ESS control and state of charge evolution within the boundaries.

In this simulation, a large error has been considered between the day ahead RES prediction $P_{d a}^{p v}$ and the real final RES output $P^{p v}$ (see Fig. 7b). In particular, far less RES energy is produced than forecasted. As a result, Fig. 7c shows that there is a gap between the planned day ahead substation power profile $P_{d a}^{g}$ and the actual one, both in the controlled cases (compare $P_{d a}^{g}$ with $P^{g, 3}$ and $P^{g, 2}$ ) and in the uncontrolled one (compare $P_{d a}^{g}$ with $P^{g, 1}$ ). Fig. 7c shows however that the proposed controller is capable of significantly smoothing the effect of the RES fluctuations on the substation flow (compare $P^{g, 1}$ with $P^{g, 3}$ and $P^{g, 2}$ ). Also in this simulation, the gray band in the figures results from 1000 experiments performed considering real short-term RES forecast affected by statistical error in line with the state of the art forecast techniques. All of the results confirm the validity of the proposed approach. Finally, Fig. 7d-e report, respectively, the ESS control and state

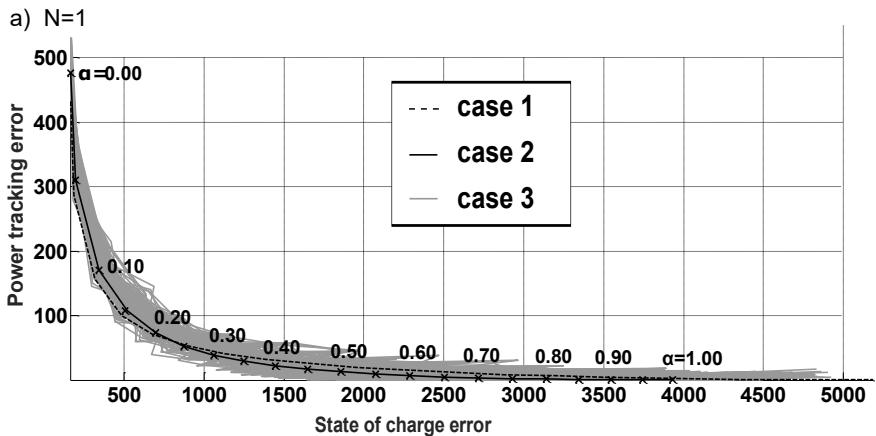

b) $\mathrm{N}=3$
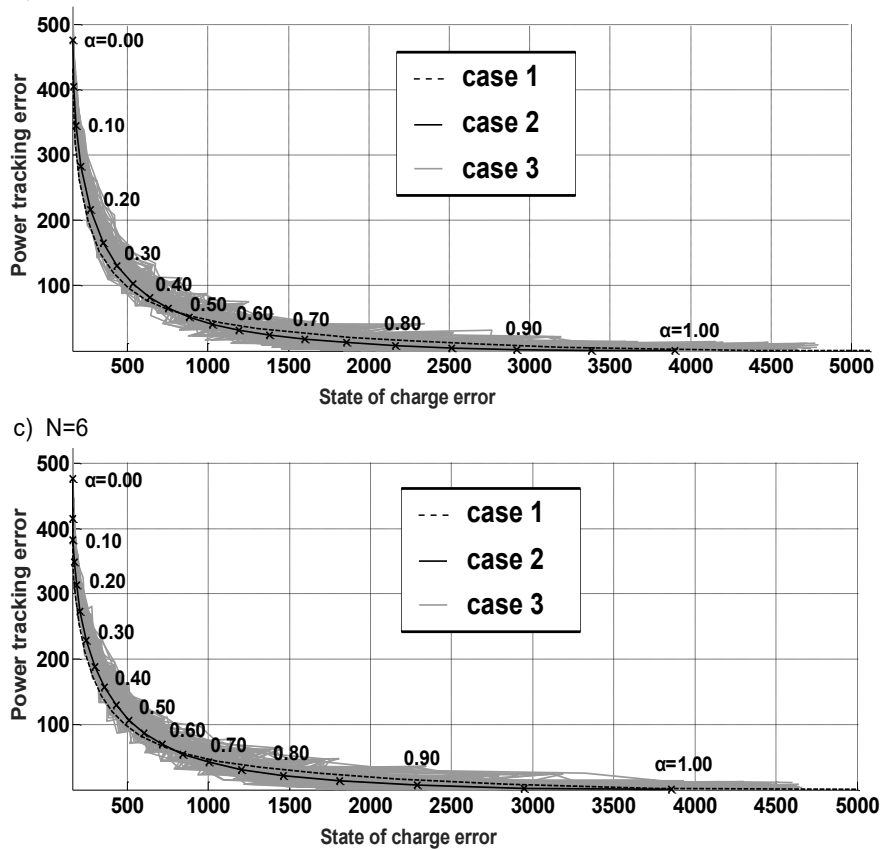

d) $\mathrm{N}=12$
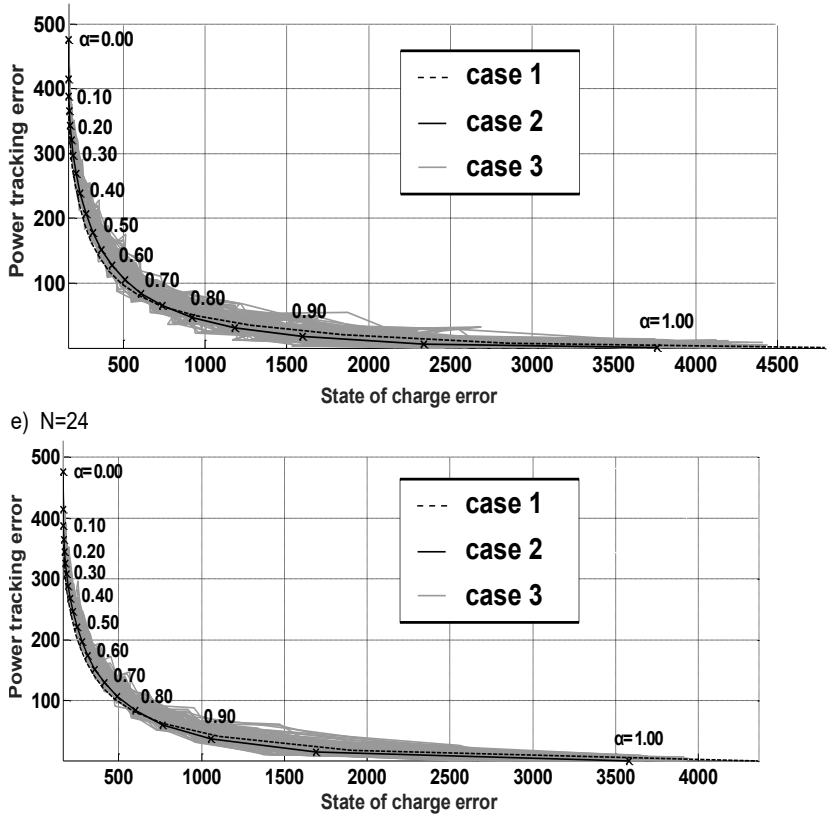

Figure 6. Simulation 2b: performance of the controller for different combinations of the target function weights and lengths of the control horizon.

of charge evolution, and show under realistic conditions that the proposed control approach enures that the storage power 

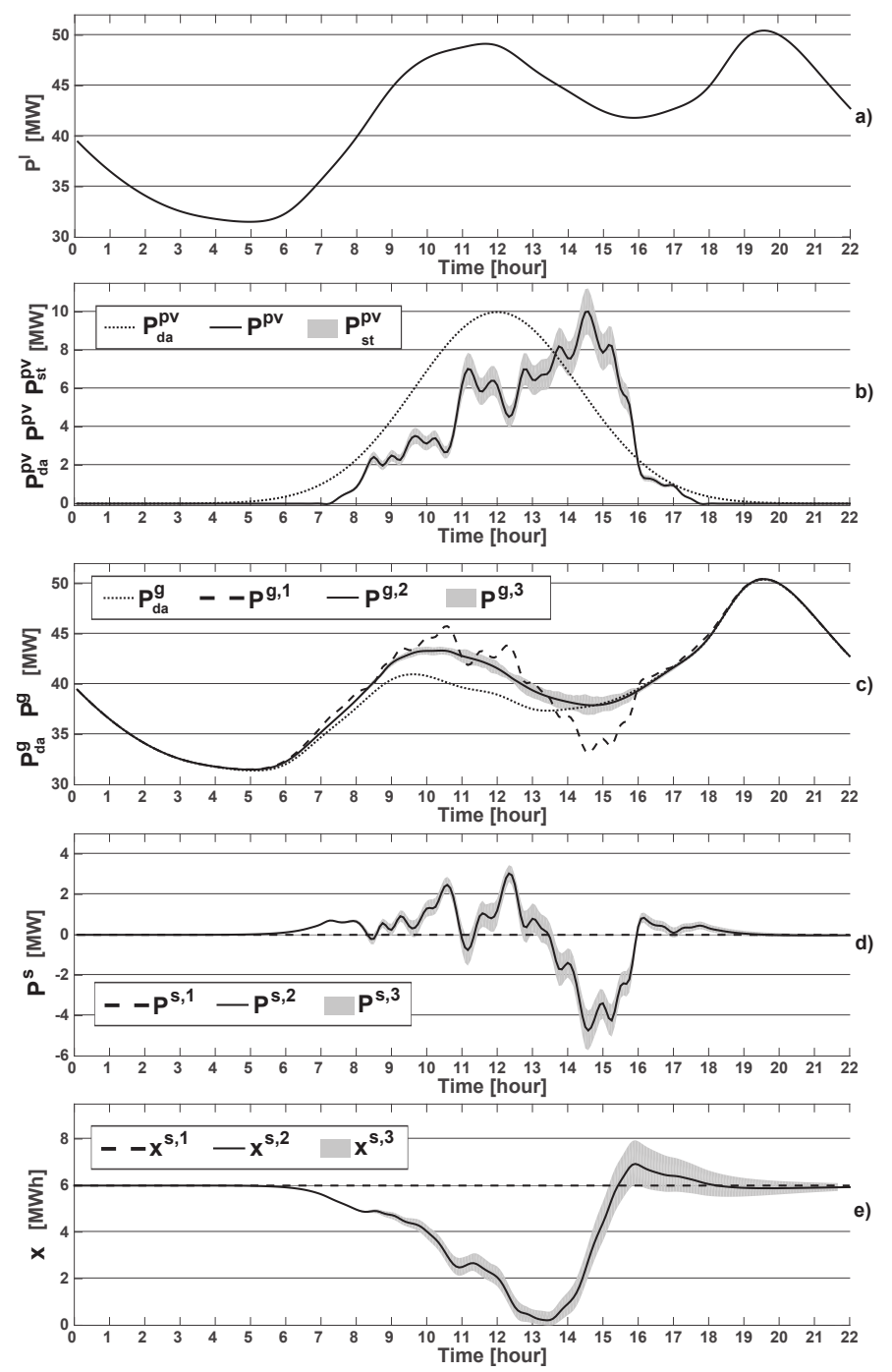

Figure 7. Simulation 3: demand (a), RES generation (b), traditional power plant power flow (c), storage active power flow (d) and storage state of charge (e).

and state of charge limits are always respected.

\section{CONCLUSiOnS}

This paper has presented a model predictive control strategy for ESS integration in an HV/MV substation operations, with the objective of controlling and smoothing the substation net power profile exchanged with the grid, in presence of high RES penetration. With the proposed control scheme, the actual power flow at HV/MV substation level can thus be controlled to track the profile agreed on the day ahead basis, while guaranteeing feasible excursions of the ESS power and state of charge. Theoretical guarantees on the stability of the proposed control scheme have been provided in a simplified setting, and a proof of the concept has been achieved in simulation scenarios characterized by both artificial test signals and real demand/generation patterns. Future works will regard the further theoretical assessment of the impact of RES imbalances on the control system performance, and the work towards field testing.

\section{ACKNOWLEDGMENT}

The authors gratefully thank the participants of the projects SAPIENZA - ATENEO 2013 and SAPIENZA - ATENEO 2014 for the helpful discussions over the matters of the paper.

\section{REFERENCES}

[1] J. von Appen, M. Braun, T. Stetz, K. Diwold, and D. Geibel, "Time in the sun: The challenge of high PV penetration in the German electric grid," IEEE Power and Energy Magazine, vol. 11, no. 2, pp. 55-64, March 2013. DOI: 10.1109/MPE.2012.2234407

[2] B. P. Roberts and C. Sandberg, "The role of energy storage in development of smart grids," Proceedings of the IEEE, vol. 99, no. 6, pp. 1139-1144, June 2011. DOI: 10.1109/JPROC.2011.2116752

[3] M. Khalid and A. Savkin, "A model predictive control approach to the problem of wind power smoothingwith controlled battery storage," Renewable Energy, vol. 35, no. 7, pp. 1520 - 1526, 2010. DOI: http://dx.doi.org/10.1016/j.renene.2009.11.030 Special Section: \{IST $\}$ National Conference 2009. [Online]. Available: http://www.sciencedirect.com/science/article/pii/S0960148109005175

[4] F. Luo, K. Meng, Z. Y. Dong, Y. Zheng, Y. Chen, and K. P. Wong, "Coordinated operational planning for wind farm with battery energy storage system," IEEE Transactions on Sustainable Energy, vol. 6, no. 1, pp. 253-262, Jan 2015. DOI: 10.1109/TSTE.2014.2367550

[5] A. Nagarajan and R. Ayyanar, "Design and strategy for the deployment of energy storage systems in a distribution feeder with penetration of renewable resources," IEEE Transactions on Sustainable Energy, vol. 6, no. 3, pp. 1085-1092, July 2015. DOI: 10.1109/TSTE.2014.2330294

[6] X. Ke, N. Lu, and C. Jin, "Control and size energy storage systems for managing energy imbalance of variable generation resources," IEEE Transactions on Sustainable Energy, vol. 6, no. 1, pp. 70-78, Jan 2015. DOI: 10.1109/TSTE.2014.2355829

[7] K. Chandy, S. Low, U. Topcu, and H. Xu, "A simple optimal power flow model with energy storage," in Decision and Control (CDC), 2010 49th IEEE Conference on, pp. 1051-1057, Dec 2010. DOI: 10.1109/CDC.2010.5718193

[8] Z. Wang, J. Zhong, D. Chen, Y. Lu, and K. Men, "A multi-period optimal power flow model including battery energy storage," in Power and Energy Society General Meeting (PES), 2013 IEEE, pp. 1-5, Jul 2013. DOI: 10.1109/PESMG.2013.6672498

[9] D. Gayme and U. Topcu, "Optimal power flow with large-scale storage integration," Power Systems, IEEE Transactions on, vol. 28, no. 2, pp. 709-717, May 2013. DOI: 10.1109/TPWRS.2012.2212286

[10] K. Nakayama, C. Zhao, L. Bic, M. Dillencourt, and J. Brouwer, "Distributed real-time power flow control with renewable integration," in Smart Grid Communications (SmartGridComm), 2013 IEEE International Conference on, pp. 516-521, Oct 2013. DOI: 10.1109/SmartGridComm.2013.6688010

[11] GRID4EU Partners, "FP7 research project GRID4EU ," Internet: http://www.grid4eu.eu/, May 2015].

[12] EnergyatHome Consortium, "Italian project EnergyatHome," Internet: http://www.energy-home.it/, May 2015].

[13] "NEC successfully commissions largest renewable Energy Storage System in Italy," Internet: http://www.nec.com, May 2015], April 2, 2014

[14] K. Worthmann, C. M. Kellett, P. Braun, L. Grüne, and S. R. Weller, "Distributed and decentralized control of residential energy systems incorporating battery storage," IEEE Transactions on Smart Grid, vol. 6 , no. 4, pp. 1914-1923, July 2015. DOI: 10.1109/TSG.2015.2392081

[15] P. Braun, L. Gruene, C. Kellett, S. Weller, and K. Worthmann, "A distributed optimization algorithm for the predictive control of smart grids," IEEE Transactions on Automatic Control, vol. PP, no. 99, pp. 1-1, 2016. DOI: 10.1109/TAC.2016.2525808

[16] A. Di Giorgio, F. Liberati, and A. Lanna, "Electric energy storage systems integration in distribution grids," in Environment and Electrical Engineering (EEEIC), 2015 IEEE 15th International Conference on, pp. 1279-1284, June 2015. DOI: 10.1109/EEEIC.2015.7165354

[17] S. Canale, F. Delli Priscoli, A. Di Giorgio, A. Lanna, A. Mercurio, M. Panfili, and A. Pietrabissa, "Resilient planning of powerline communications networks over medium voltage distribution grids," in Control Automation (MED), 2012 20th Mediterranean Conference on, pp. 710715, July 2012. DOI: 10.1109/MED.2012.6265721

[18] D. Mayne, J. Rawlings, C. Rao, and P. Scokaert, "Constrained model predictive control: Stability and optimality," Automatica, vol. 36, no. 6, pp. 789-814, 2000. DOI: 10.1016/S0005-1098(99)00214-9 
[19] G. Di Pillo and F. Facchinei, "Exact barrier function methods for Lipschitz programs," Applied Mathematics and Optimization, vol. 32, no. 1, pp. 1-31, 1995. DOI: 10.1007/BF01189901

[20] M. D. Felice, M. Petitta, and P. M. Ruti, "Short-term predictability of photovoltaic production over Italy," Renewable Energy, vol. 80, pp. 197 - 204, 2015. DOI: 10.1016/j.renene.2015.02.010. [Online]. Available: http://www.sciencedirect.com/science/article/pii/S0960148115001007

[21] B. Wolff, E. Lorenz, and O. Kramer, "Statistical learning for short-term photovoltaic power predictions," in Proceedings of DARE 2013 Workshop on Data Analytics for Renewable Energy Integration, September, vol. $23,2013$. 